Revue internationale P.M.E.

Économie et gestion de la petite et moyenne entreprise

\title{
La formation professionnelle continue dans les PME européennes : enjeux et problématiques à travers quatre études de cas
}

\section{Philippe Sarnin}

Volume 11, numéro 2-3, 1998

URI : https://id.erudit.org/iderudit/1009047ar

DOI : https://doi.org/10.7202/1009047ar

Aller au sommaire du numéro

Éditeur(s)

Presses de l’Université du Québec

ISSN

0776-5436 (imprimé)

1918-9699 (numérique)

Découvrir la revue

Citer cette note

Sarnin, P. (1998). La formation professionnelle continue dans les PME européennes : enjeux et problématiques à travers quatre études de cas. Revue internationale P.M.E., 11(2-3), 127-159. https://doi.org/10.7202/1009047ar
Résumé de l'article

Cet article prend appui sur une recherche internationale réalisée pour le compte de la Commission européenne dans le cadre du programme "FORCE "

Cette recherche répond à deux finalités principales :

- repérer les pratiques innovantes de formation en PME qui favorisent le développement des qualifications et de remployabilité des salariés,

- repérer et analyser les mécanismes en jeu (acteurs externes et internes, facteurs de réussite des actions de formation, contextes économiques et institutionnels, situations concrètes d'application).

Les cas présentés permettent une clarification du contexte et des problématiques de la formation continue de quatre entreprises de quatre pays différents dans le secteur agroalimentaire.

Le modèle de comparaison utilisé comprend six niveaux d'analyse :

- le contexte de l'entreprise,

- les problématiques dominantes du cas,

- les modes de résolution des problèmes de formation continue,

- la conception de la formation professionnelle,

- la méthodologie employée,

- les possibilités de transferí des pratiques innovantes. 


\title{
La formation professionnelle continue dans les PME européennes: enjeux et problématiques à travers quatre études de $\operatorname{cas}^{1}$
}

Philippe SARNIN

Université Aix-Marseille II

\author{
MOTS CLÉS
}

\section{Formation continue - PME - Europe - Agroalimentaire Qualité - Qualification}

\begin{abstract}
RÉSUMÉ
Cet article prend appui sur une recherche internationale réalisée pour le compte de la Commission européenne dans le cadre du programme "FORCE". Cette recherche répond à deux finalités principales:

- repérer les pratiques innovantes de formation en PME qui favorisent le développement des qualifications et de l'employabilité des salariés,

- repérer et analyser les mécanismes en jeu (acteurs externes et internes, facteurs de réussite des actions de formation, contextes économiques et institutionnels, situations concrètes d'application).
\end{abstract}

Les cas présentés permettent une clarification du contexte et des problématiques de la formation continue de quatre entreprises de quatre pays différents dans le secteur agroalimentaire.

Le modèle de comparaison utilisé comprend six niveaux d'analyse:

- le contexte de l'entreprise,

- les problématiques dominantes du cas,

- les modes de résolution des problèmes de formation continue,

- la conception de la formation professionnelle,

1. Cet article résume le rapport réalisé par Deirdre Hunt (Cork University), Philippe Poirson (Groupe ESC Lyon), Philippe Sarnin (Université Aix-Marseille II, rapport réalisé dans le cadre de l'IRE-Groupe ESC Lyon) : «Études de cas européennes concernant la formation continue dans l'industrie agro-alimentaire », Programme communautaire «FORCE», Convention No 94-23-SEC-0053-00, mai 1995. Le travail empirique a été réalisé avec l'aide de R.D. King et J.A. Burns de l'Université de Reading, Ruggiera Sarcina de l'Instituto Guglielmo Tagliacarne et Jacqui Spence de l'ILRES (Luxembourg). 
- la méthodologie employée,

- les possibilités de transfert des pratiques innovantes.

\begin{abstract}
Based on a research project realized for the program "Force" funded by the European Commission, this article has two main objectives:

- to examine innovative training practices facilitating the developement of qualifications and employability for the workers in medium sized firms;

- to analyse some issues such as the role of inside and outside actors, the success factors for continued vocational education, the influence of the socio-economic context and the concrete resolution adopted by firms.
\end{abstract}

The four cases belong to the food and drink industry. The firms are localized in Italy, Luxemburg, Ireland and United Kingdom. They were studied in six dimensions:

- the context of the firm

- the dominant issues of the case

- the way of resolution adopted

- the conception of vocational training in play

- the methodology used

- the possibilities for transfering innovative practices.

\title{
RESUMEN
}

Este artículo se apoya sobre una encuesta internacional realizada por la Comisión Europea dentro del programa "Force". Esta encuesta responde a dos objetivos:

- localizar las prácticas innovadoras de formación en las empresas de pequeña y media importancia que favorecen el desarrollo de la calificación y del empleo de los salariados;

- localizar y analizar los mecanismos puestos en juego.

Los casos presentados permiten una clasificación de los diferentes contextos y de la problemática encontrada sobre la formación continua de cuatro empresas de cuatro paises diferentes en el sector agro-alimentario.

\section{L'AUTEUR}

Philippe Sarnin détient les diplômes suivants: DESS de psychologie clinique, option clinique de la formation (Lyon II, 1986), diplôme de l'Université d'informatique appliquée aux sciences sociales (Lyon II, 1986), DEA de démogaphie et sciences sociales appliquées (Lyon II, 1987), doctorat de I'Université de sociologie et sciences sociales sous la direction de Philippe Bernoux (Lyon II, 1993)

Il est actuellement maître de conférences en psychologie du travail et des organisations (depuis septembre 1995) et chargé d'enseignement dans les universités Lyon II, Lyon III, Paris I et à l'INSA de Lyon.

Auparavant, il a été professeur et responsable de recherche au groupe ESC Lyon - Institut de recherche de l'entreprise, de septembre 1986 à août 1995.

Adresse: Université de la Méditerranée - Aix-Marseille II, Département hygiène, sécurité, environnement, 163, avenue de Luminy, Case 909, 13288 Marseille Cedex 9, Téléphone : 04918297 36, Télécopieur : 04918297 40. Courriel : sarnin @luminy.univ-mrs.fr 


\section{Introduction}

Les transformations importantes de l'industrie européenne reposent pour une large part sur les changements technologiques qui la traversent et sur l'évolution des systèmes concurrentiels. Comprendre les pratiques de travail les plus appropriées à ces modifications devient un enjeu socio-économique majeur. La Commission européenne (Task Force for Human Resources) a lancé une série d'études pour analyser comment dans plusieurs grands secteurs industriels les pratiques de formation continue permettent de répondre à cet environnement délicat.

Nous disposons depuis 1993 de travaux innovants ayant permis de repérer des pratiques intéressantes dans des secteurs autrefois ignorés. En particulier, le secteur des industries agroalimentaires ${ }^{2}$, largement développé dans tous les pays et reposant pour une grande partie sur de nombreuses PME, a été étudié en détail. Les précédentes recherches montrent en effet un certain nombre de constantes à travers les pays de la Communauté bien que tous n'aient pas les mêmes moyens de réagir à ces bouleversements.

- Dans tous les pays existe une industrie agroalimentaire souvent composée en très large majorité d'entreprises de petites dimensions. Ce qui n'est pas le cas d'industries comme l'automobile ou la machine-outil, bien que ces dernières soient très connues et fassent l'objet de nombreuses recherches.

- Les investissements technologiques y sont très importants, portant à la fois sur les capacités de production et sur la régularité et la qualité des produits.

- Les produits alimentaires sont très marqués culturellement. Ils concernent alors des marchés locaux. Mais ils peuvent aussi avoir du succès au niveau international, ce qui pose aux entreprises des problèmes souvent très complexes de commercialisation, de normes d'hygiène, de transport, etc.

2. À la fin des années 1980 , l'industrie emploie dans l'Europe des 12 plus de 3,2 millions de personnes pour un chiffre d'affaires de plus de 620 milliards d'ÉCU. Au moins $60 \%$ de la production est réalisée par la France, l'Allemagne et le Royaume-Uni, cependant qu'une forte croissance est constatée dans ce secteur en Irlande, en Espagne, en Italie et au Portugal. Globalement, l'emploi est à $70 \%$ localisé en Allemagne, en Espagne, au Royaume-Uni et en France. Entre 1980 et 1990, les effectifs ont baissé de $11 \%$ dans ce secteur sur l'ensemble de la Communauté européenne.

$\mathrm{Au}$ niveau européen, cette industrie représente $15 \%$ de la valeur ajoutée industrielle. Si la part globale des industries agroalimentaires diminue lorsque l'économie d'un pays se développe fortement, on constate aussi une évolution nette vers une transformation plus forte des produits avec, chez les consommateurs, une demande croissante de produits très préparés. Pour certains pays européens, l'agroalimentaire représente aussi une part importante des exportations : près de $50 \%$ pour l'Irlande, entre 30 et $35 \%$ pour le Danemark, les Pays-Bas ou la Belgique. Les entreprises de ce secteur sont, d'après les données Eurostat, au nombre de 264000 en 1988, dont $92 \%$ emploient moins de 20 personnes. 
- Les problèmes d'hygiène et de sécurité sanitaire des productions sont des enjeux clés dans l'ensemble de ce secteur. Les clients ne pardonnent pas les écarts sur ce plan et l'ensemble des producteurs, avec l'aide des directives de la Commission européenne, font actuellement un effort considérable qui touche d'ailleurs particulièrement les transformateurs de produits alimentaires. Cet enjeu est la forme spécifique que prend dans ce secteur le problème plus général de la qualité.

- Comme dans beaucoup de secteurs, on constate également, et cela est aussi lié aux enjeux précédents, des politiques visant à réduire le nombre de niveaux hiérarchiques, à favoriser la prise de décision là où elle se fait avec pertinence, c'est-à-dire à donner plus de responsabilités, d'autonomie à des salariés de base.

- La recherche de flexibilité, sous ses différentes formes, est aussi à l'ordre du jour dans cette industrie. Les phénomènes de mode ou la recherche de différenciation et de produits nouveaux, l'adaptation à des contextes culturels spécifiques, les problèmes de conservation des aliments figurent parmi les facteurs à l'origine de cette tendance ${ }^{3}$.

Dans chacun de ces domaines, la formation permanente des salariés est une condition de réussite de ces politiques, mais aussi un élément important du maintien de «l'employabilité » de ceux-ci à travers un effort qui peut apparaître considérable pour certains. Les travaux existants permettent de se rendre compte de ces changements, de la place capitale qu'y joue la formation professionnelle continue et qui conduit les entreprises à essayer de se transformer en « organisations apprenantes » ne pouvant pas se contenter de vivre sur leurs acquis ou dans une niche de marché souvent fragile.

3. Les préoccupations relatives à la santé, à la forme, ont conduit à des transformations fortes des produits de base débouchant sur des tendances complexes : avoir des produits «naturels» tout en étant pratiques à utiliser, prêts à consommer, pose des problèmes nouveaux aux industriels en plus de renforcer l'enjeu de l'hygiène.

Deux tendances contradictoires se manifestent également. D'une part, une certaine uniformisation des modes alimentaires à travers la diffusion de la « restauration-minute », voire des cuisines « ethniques» (chinoise, mexicaine, italienne, etc.). D'autre part, une augmentation de la variété des produits disponibles et le maintien, voire le développement de produits locaux, parfois fabriqués de manière artisanale, ainsi que la restauration de loisirs, de plaisir (slow-food) coexistent avec la tendance précédente à la large diffusion de certains produits. Chez les producteurs, cela implique une variété importante de produits, la combinaison d'économies d'échelle avec des « savoir-faire » ou des références de type artisanal. Les conséquences sont alors de développer la flexibilité des productions, la qualité des processus... Donc, là aussi, le développement des qualifications devient un enjeu important. 


\section{La formation continue dans les PME}

La formation professionnelle est un enjeu capital pour les PME. Les spécificités de ces entreprises font que les pratiques de formation sont au cœur du succès ou des échecs des politiques de changement. La division du travail est souvent moindre en PME et les salariés sont plus polyvalents. Plus l'entreprise est petite, plus le lien est étroit entre les « compétences stratégiques » de la firme et les compétences professionnelles des travailleurs (Sarnin, 1990). En conséquence, chaque programme de formation mis en œuvre représente un changement significatif pour l'organisation.

Malgré cela, de nombreux auteurs ${ }^{4}$ soulignent les difficultés des PME à développer des programmes de formation professionnelle conséquents. Les problèmes posés sont très souvent les suivants :

- en comparaison avec les grandes entreprises, les dépenses des PME consacrées à la formation sous forme de sessions classiques assurées par des organismes de formation sont faibles. Le fait que les travailleurs sont très polyvalents signifie aussi que leurs compétences sont souvent construites «sur le tas»;

- quand la main-d'œuvre est en nombre limité, il devient très problématique d'envoyer un employé à l'extérieur pour une ou plusieurs semaines. Cela peut complètement bloquer le processus de production. De nombreuses enquêtes soulignent également que les écarts existant dans l'accès à la formation selon le niveau hiérarchique sont accentués dans les PME;

- le rôle des PME est important pour la formation et la socialisation professionnelles. Mais, à cause des possibilités limitées pour offrir des carrières significatives aux travailleurs, beaucoup d'entre eux préfèrent aller dans une plus grande entreprise après s'être formés et avoir obtenu une qualification reconnue. Ce qui gêne de nombreux patrons de PME qui hésitent devant ce qui peut apparaître comme un investissement à fonds perdus ;

- les stratégies à court terme que suivent les PME et qui sont liées aux contraintes qui pèsent sur leur autonomie rendent difficile la mise en place de programmes à long terme dans le domaine de la formation. Comment planifier une formation de trois ans pour un travailleur alors qu'on ne connaît pas le niveau d'activités dans trois mois?

La formation professionnelle en PME est bâtie sur un paradoxe : beaucoup de facteurs entravent son développement et, en même temps, elle constitue un élément clé pour la pérennité et la croissance de ce type d'entreprises. Notre principale

4. Voir Lutz (1984), par exemple. 
hypothèse, pour faire face à ce paradoxe, est que la formation professionnelle peut prendre des formes efficaces et innovantes qui ne sont pas très visibles si l'on pense avec les « lunettes » des grandes entreprises ou avec les concepts et les catégories construits pour analyser les problèmes de formation à un niveau macrosocial. Les cas présentés dans ce texte exemplifient ces formes.

La variété des contextes institutionnels en Europe semble impliquer l'existence d'enjeux différents pour les entreprises. En fait, quand nous observons ce qui se passe dans un même secteur et pour un même type d'entreprises, les différences ne sont pas si marquées. Du fait de la similarité des tendances en matière de technologie, de changement des marchés ou d'application des directives de la Commission, par exemple, beaucoup d'enjeux sont identiques à travers l'Europe dans l'industrie agroalimentaire, tels que la qualité, la certification des procédés, l'hygiène, le développement de zones rurales ou pauvres, etc. La principale différence tient aux ressources, qualitatives et quantitatives, disponibles dans chaque pays à travers les organisations existantes et les politiques nationales de formation mises en œuvre.

\section{La démarche suivie}

Ce travail s'appuie sur la série d'études sectorielles menées à l'initiative de la Commission européenne qui mettait en évidence les problématiques relatives à la formation continue.

Dans le secteur agroalimentaire, les travaux réalisés se sont avérés très riches, reposant en particulier sur des études de cas qui permettent de comprendre de manière très concrète les enjeux des entreprises et les solutions innovantes développées en matière de formation continue.

Parmi les problèmes qui peuvent être abordés à travers ces études de cas, on peut citer sans être exhaustif les questions suivantes:

- Quels sont les dispositifs les plus innovants et efficients en matière de formation continue?

- Quels sont les facteurs clés de succès?

- Quels rôles doivent être joués par les acteurs internes et externes (managers, partenaires sociaux, organismes de formation ou de soutien publics ou privés)?

- Quels éléments innovants peuvent être transférés ? À quelles conditions?

- Quelle place joue la formation dans la formulation et la mise en œuvre des stratégies des entreprises? 
- Comment la formation continue permet-elle à la fois de maintenir, voire de développer l'avantage concurrentiel de l'entreprise tout en sauvegardant l'employabilité des salariés?

La démarche suivie pour réaliser ces cas a consisté à interroger un échantillon d'acteurs dans quatre entreprises de l'agroalimentaire en Italie, au Luxembourg, en Irlande et en Grande-Bretagne.

Nous avons rencontré à chaque fois de huit à 20 personnes : managers, responsables de la formation, des ressources humaines, direction générale, employés, ouvriers, partenaires sociaux, selon leur implication dans les dispositifs de formation mis en œuvre.

De plus, différents documents ont été collectés auprès de l'entreprise, mais aussi auprès d'organismes extérieurs sur les contextes institutionnels locaux et nationaux de la formation.

Notre préoccupation a été de recueillir suffisamment d'éléments pour couvrir les domaines suivants :

- l'entreprise : produits, processus de production, marchés, historique, stratégie;

- les pratiques de formation habituelles et les pratiques innovantes;

- les conditions de réussite;

- la place de la formation par rapport à la stratégie de l'entreprise et son articulation avec les autres éléments de gestion du personnel;

- le rôle des différents acteurs ;

- l'implication des partenaires sociaux;

- les contraintes et les ressources du contexte constitutionnel : législation, conventions collectives, organismes disponibles, etc. ;

- les évaluations réalisées des dispositifs;

- les opinions des différents acteurs par rapport aux pratiques, leurs souhaits pour le futur;

- les politiques en matière d'innovation, de qualité, d'hygiène.

Les quatre entreprises qui font l'objet de ces études de cas ont été choisies principalement en fonction de leur réussite dans la mise en place de démarches de formation continue innovantes. Nous nous sommes appuyé sur les travaux précédemment réalisés pour l'étude sectorielle du secteur agroalimentaire en 1993, dans le cadre du programme FORCE, ainsi que sur les conseils des chercheurs ayant réalisé ces travaux. 


\section{Les entreprises et leurs pratiques de formation}

\subsection{Luxfood (Luxembourg)}

Luxfood s'occupe plus particulièrement de la transformation de la viande au sein d'une entreprise coopérative agroalimentaire plus vaste créée au lendemain de la Seconde Guerre mondiale. L'usine permet toutes les opérations de transformation des viandes depuis l'abattage des animaux jusqu'à l'emballage des produits traités prêts pour l'expédition. Orientée sur la production, l'entreprise est constituée d'une quinzaine de sections employant 160 personnes et comprenant chacune un chef de section assisté d'une équipe d'ouvriers et d'ouvrières plus ou moins polyvalents. L'abattage des animaux n'est en effet pas permanent, aussi le rythme d'une journée, pour les ouvriers au début de la chaîne d'opérations, correspond à un accompagnement ordonné de la viande : certains passent ainsi de l'abattage à la découpe, d'autres alternent entre les divers traitements.

La gamme des produits fabriqués comprend environ 600 références destinées aux particuliers sous différentes marques, mais aussi pour les professionnels : bouchers, restaurants, hôtels et distribution. Les animaux abattus et découpés sont des porcins, des bovins et des ovins. Toute en longueur, l'usine est organisée en fonction du cycle de production : les animaux arrivent d'abord dans des étables à une extrémité du bâtiment et les produits finis en sortent à l'autre extrémité où une boutique de vente pour les visiteurs est d'ailleurs installée.

D'une manière générale, les qualifications des opérateurs sont surtout élevées chez ceux qui assurent la découpe des viandes. De même, les hommes sont majoritaires au début du processus alors qu'après la découpe ce sont plutôt des femmes, sauf pour la section «grande cuisine».

Les machines automatiques utilisées concernent surtout la fin du processus, car le travail de découpe est essentiellement effectué à la main par des ouvriers. Chaque animal étant différent et les lignes de coupe étant précises, on ne peut qu'améliorer les outils (scies, couteaux) et utiliser le savoir-faire des ouvriers.

L'entreprise emploie également une proportion importante de travailleurs étrangers, frontaliers mais aussi d'origines géographiques plus éloignées. Seize nationalités différentes sont représentées et $70 \%$ du personnel d'exécution non qualifié vient de l'étranger sans pour autant être citoyen des pays frontaliers du Luxembourg. La transformation de la viande intéresse peu les nationaux qui préfèrent, par exemple, s'orienter vers les activités tertiaires ou la fonction publique, et n'attire pas les travailleurs allemands, belges ou français. Luxfood emploie principalement des travailleurs immigrés d'origine portugaise, population qui était accessible au moment du développement de l'entreprise tout en n'étant pas trop éloignée des pratiques culinaires correspondant aux produits de l'entreprise. 
Parmi les opérateurs intervenant directement dans le processus de production, on compte une vingtaine de personnes ayant la qualification de "maitre-boucher », une soixantaine possèdent un $\mathrm{CATP}^{5}$, les autres n'ont pas de qualification spécifique liée à un diplôme.

La direction de l'entreprise souligne le fort accroissement de contraintes supplémentaires pesant sur l'entreprise :

On est forcément amené à restructurer [...] je suppose que toutes les entreprises sont dans ce cas parce que les directives, mais pas dans le sens des directives communautaires, mais les pressions, les législations respectives, les demandes qui viennent de l'extérieur et qui retombent sur les entreprises sont devenues telles que les gens sont forcés d'utiliser toute leur énergie pour le service, la rentabilité des entreprises. Je m'explique : avant, c'est l'État, c'est la santé qui prenait ça en charge [...] Maintenant, vu que les États ou les directives disent que ce n'est pas le rôle des États de contrôler les entreprises, elles doivent se contrôler elles-mêmes. Maintenant, c'est une directive, ça veut dire qu'on doit créer un laboratoire de contrôle, soit on doit aller acheter ses services ailleurs. On dit «vous devez faire de la formation», [...] or au Grand Duché $[\ldots]$ la fonction de désosseur ça n'existe pas dans les structures de formation, la formation des gens retombe de nouveau sur l'entreprise [...] Puis il y a l'environnement. Subitement on dit «l'eau doit être plus propre », « on doit créer une station biologique ». Qui paie ? Les entreprises, des coûts supplémentaires [...] Maintenant, c'est la santé au travail [...] éviter que quelqu'un devienne malade [...] ça pourrait être fait dans les écoles d'une façon plus conséquente, non c'est dans les entreprises puisque c'est dans les entreprises qu'on a l'argent [...] puis aussi il faut subvenir aux dépenses sociales, aux caisses de maladie... [...] les entreprises sont forcées d'utiliser toute leur énergie pour faire face à ces dépenses supplémentaires. Il y a un élément supplémentaire, c'est que les frontières étant ouvertes depuis un ou deux ans, la concurrence se renforce durablement... (Cadre supérieur)

Le poids de ces contraintes apparaît accentué pour les entreprises comme Luxfood par rapport aux autres opérateurs de la filière. En amont au niveau des fermes et en aval pour le transport et les petits distributeurs, les contrôles qualité sont moins denses. Ce qui rend d'ailleurs les techniciens-qualité de l'entreprise un peu amers : tous leurs efforts et les dépenses afférentes peuvent être réduits à néant par un petit épicier qui assure mal la chaîne du froid.

Globalement, l'entreprise n'a pas eu d'accident majeur en matière d'hygiène. Cependant, il s'agit d'un enjeu permanent et dont la réussite repose majoritairement sur les opérateurs, leur formation et leur conscience professionnelle. Pour la formation professionnelle, il ne s'agit donc pas seulement d'acquérir des connaissances à ce sujet ou même des savoir-faire mais aussi d'amener une prise de conscience

5. CATP : Certificat d'aptitudes techniques et professionnelles, diplôme final du régime professionnel (en apprentissage) de l'enseignement secondaire au Luxembourg. La «maîtrise » dans un même domaine demande une année d'études supplémentaire. 
suffisante de cet enjeu pour que chaque salarié ait une attitude constante d'hygiène dans le moindre de ses comportements. La normalisation des procédés de fabrication par le biais des certifications ISO, la multiplication des mesures effectuées ne peuvent enlever le fait que ces attitudes et surtout leur constance sont l'élément clé de réussite en ce domaine. Actuellement, l'entreprise engage un processus lourd de mise en place de systèmes de contrôle des aliments pour se soumettre à la directive de la Commission européenne prônant l'usage de méthodes de type «HACCP » (Hazard Analysis and Critical Control Points) :

Ça consiste à décrire le produit en détail avec toutes les variations de la viande. Il faut décrire aussi tout le processus et les matières premières. On détermine les points critiques en fonction d'un arbre de décision en 11 étapes par rapport aux risques de contamination. Puis on regarde les mesures à prendre sur ces points critiques pour les maîtriser : ça peut aller jusqu'à changer de machine, de procédés ou de produit. (Responsable qualité)

Une première opération HACCP concerne les pâtés de foie. À terme, 400 produits devront être examinés de la même manière même si les responsables de l'entreprise pensent que l'autocontrôle en cours est déjà satisfaisant.

La chance qu'on a, c'est d'avoir déjà un laboratoire, mais tout est important. Chaque cuisson est analysée, on sait déjà les problèmes quand il y a des corps étrangers : cheveux, laque à ongle. Ça va conduire à accroître encore le nombre de mesures de contrôle et aussi plus de responsabilités pour les ouvrières qui auront à en faire. On a déjà admis depuis longtemps qu'un ouvrier ait le droit d'arrêter la production lui-même s'il constate un problème. (Responsable qualité)

La formation continue dans cette entreprise prend plusieurs formes :

- au niveau de l'Agrocentre, le département personnel gère une part importante du développement des formations techniques et managériales. Un groupe projet, par exemple, réfléchit et fait des recommandations sur les objectifs de formation nécessaires pour répondre aux diverses directives européennes et pour atteindre les certifications ISO;

- l'Agrocentre met également à disposition des salariés 70 abonnements à des revues et magazines spécialisés dans les diverses activités du site ;

- les formations externes sont assurées principalement par les organisations luxembourgeoises suivantes : Chambre de commerce, Chambre des employés privés, Bureau pour l'accroissement de la productivité et par des centres spécialisés étrangers (France, Allemagne, Belgique, Suède...). Au niveau de l'Agrocentre, le budget annuel de formation varie entre 20000 et 40000 ÉCU. Il n'y a pas à proprement parler de minimum ni de maximum fixés pour ces dépenses. Tout dépend des besoins identifiés ; 
- la formation interne est en particulier assurée par les personnes ayant suivi une formation à l'extérieur, car l'entreprise ne dispose pas de formateurs permanents. De même, il est habituel de considérer que chaque responsable a un rôle formateur vis-à-vis de ses collaborateurs, y compris au niveau des chefs de section.

Pour l'ensemble de l'Agrocentre, on constate que c'est Luxfood qui consomme le plus de formations externes : en 1991, par exemple, 29 actions peuvent être identifiées à Luxfood sur 49 pour l'ensemble de l'Agrocentre. Ces différentes actions n'ont cependant pas toutes la forme de séminaires. Il s'agit en effet majoritairement de participations à des conférences, congrès, salons, foires, portes ouvertes. Dix actions sur les 29 de 1991 correspondent réellement à des séminaires de formation à proprement parler. Les participants de ces actions sont majoritairement membres de l'encadrement technique de Luxfood. Plus de $80 \%$ de ces actions ont lieu en dehors du Luxembourg. Les séminaires portent, par exemple, sur la cuisson du jambon, l'autoclavage des conserves, la stérilisation, etc. Pour les opérateurs, la première formation pour les postes peu qualifiés est donnée par le chef de section.

Les chefs de section détiennent en général un diplôme de maîtrise professionnelle et sont spécialisés dans ce métier. Huit d'entre eux ont été envoyés en Suède pour un stage dans une fabrique similaire au démarrage de Luxfood.

En 1986, l'entreprise avait embauché des ouvriers en les faisant travailler en doublon avec un boucher pour apprendre les techniques du désossage et de la découpe. De même, deux apprentis ont travaillé au cours de leur formation dans l'entreprise il y a trois ans. Cependant, il n'est pas facile de les conserver après que l'entreprise a fait l'effort de les former:

Il y en a plusieurs qui sont allés travailler sur leur commune [...] Il faut dire quelque chose, le travail chez nous ce sont en plus des travaux qui sont relativement pénibles, surtout si on prend la découpe : vous êtes toute la journée à $12^{\circ} \mathrm{C}$ maximum et ça, debout sur les jambes. (Responsable de production)

Au quotidien, la formation sur le tas et l'information des salariés passent par d'autres voies :

Chaque jour, on a une réunion avec les responsables où il y a quelqu'un du service technique qui participe et là, on fait le tour de table des problèmes journaliers. S'il y a une chose spécifique concernant surtout une section, on se met ensemble avec ce gars pour analyser les problèmes et trouver des solutions avec lui ; il y a aussi le programme de production pour chaque section. (Responsable de production)

Pour ce qui est des opérateurs, il revient également au chef de section d'apporter des éléments d'information. De même, ils ont pour mission d'expliquer l'utilisation des outils et les mesures d'hygiène et de sécurité (tabliers et gants en acier pour la découpe). Cependant, les cadres de l'entreprise n'attendent pas des 
chefs de section qu'ils diffusent des informations trop au-delà de leur cadre de travail :

Dans les réunions où les chefs de section se mettent ensemble avec les ouvriers, c'est pas souvent, il faut pas trop en raconter non plus parce que s'ils disent de trop, le lendemain, c'est dans les cafés.

Il y a aussi le problème de la compréhension, donc certains comprendront exactement le contraire de ce que vous voulez dire.

Pour essayer de faire comprendre les problèmes de qualité et d'hygiène dans la manipulation de produits alimentaires, des cours de chimie et de biologie ont été mis en place auprès des chefs de section et des personnels peu qualifiés du laboratoire. Plutôt que de les envoyer suivre un stage à l'extérieur, c'est le responsable du laboratoire, biologiste de formation, qui a organisé une formation interne sur le lieu de travail. Il n'était pas évident de faire un cours universitaire, aussi le rôle de ce responsable a été capital dans la réalisation du cours :

Pour le cours de chimie et biologie, j'ai tout créé moi-même, et sur la stérilisation/ pasteurisation, c'était suite à un stage que j'ai fait en Allemagne. Disons que c'est un cours que j'ai dû transformer. J'ai pris mon livre et j'ai transformé. Quand vous faites un cours, c'est après les heures de travail, les gens avaient déjà 8 ou 9 heures de travail et donc il faut rendre ça intéressant, donc il faut prendre plein d'exemples qui rentrent dans leur métier pour que ça accroche.

Ces cours duraient de une à deux heures en fin de journée avec une phase importante de discussion après l'exposé ou la démonstration. Une quinzaine de séances ont été organisées en biologie et une vingtaine en chimie. Les formés étaient, selon la direction, « semi-volontaires ». Le premier cours a accueilli neuf personnes et quinze, pour celui de chimie.

Pour le responsable du laboratoire, cette expérience s'est avérée à la fois riche et réussie :

J'avais un programme, mais avec les discussions les gens posaient n'importe quelle question. C'était très vivant, c'est plus rentable comme ça, en même temps ils acceptent plus facilement la théorie [...] Les questions portaient à la fois sur le travail et la vie privée. En macrobiologie, il y a plein de problèmes qu'on rencontre dans le privé parce que c'est un domaine qui nous concerne tous, car nous sommes également consommateurs. Par exemple, pour certaines personnes, c'est tout à fait automatique de se laver les mains et pour d'autres, c'est pas le cas [...]

On regardait les bactéries au microscope, on faisait des colorations. Je leur demandais de comparer les bactéries qu'ils avaient dans les mains : même après les avoir lavées, il en reste quand même. Ça les a familiarisés aussi avec les techniques du laboratoire [...] Pour le cours de chimie, c'était plus difficile, les gens ont plus peur, c'est plus abstrait. Par exemple, le tableau de Mendeleïev c'est long à expliquer, c'est plus loin de la vie quotidienne alors qu'en production, on utilise beaucoup de produits chimiques. 


\subsection{David Hall Ltd (Royaume-Uni)}

Hall est une entreprise spécialisée dans la fabrication et la préparation du jambon et du lard et est implantée dans une région où la consommation de ce produit est faible par rapport au reste de la Grande-Bretagne. Cependant, comme l'indiquent les tendances de consommation en Grande-Bretagne, la consommation de produits à base de viandes et de plats préparés est, dans la région, supérieure à la moyenne. Étant donné que Hall doit être concurrentielle pour garder sa position sur le marché, l'entreprise élabore sa gamme de produits à valeur ajoutée en adéquation avec les tendances des marchés locaux. Environ 50\% des ventes de l'entreprise sont réalisées sous la marque déposée « Hall » et sont commercialisées à de petits détaillants en Écosse ainsi que dans le Nord de l'Angleterre à travers sept centres commerciaux. Le reste de la production est vendu directement à des chaînes de distribution.

David Hall Ltd est une entreprise privée composée en grande partie des membres de la famille Hall impliqués dans la gestion courante de l'entreprise. Ils siègent également au conseil d'administration. En Écosse, Hall est le plus grand fabricant indépendant de jambon et de lard, de viandes cuites et de saucisses. L'entreprise achète et abat environ 4000 cochons par semaine, elle produit toute une gamme de viandes salées, de pâtisseries salées, d'épicerie fine, de viandes cuites selon des recettes traditionnelles.

L'entreprise emploie 1350 personnes et est située dans une région où l'on trouve facilement de la main-d'œuvre, bien que les personnes employées aient, en général, peu suivi de formation initiale. En effet, peu d'employés disposent de qualifications reconnues. Les salaires sont en général situés légèrement au-dessus de la moyenne pour la région, cela principalement pour compenser l'environnement peu attrayant qu'offre une usine de transformation des viandes.

Dans l'organisation de l'entreprise, la position d'un employé sur la chaîne de fabrication est indiquée par des casquettes de couleur portées par les employés. Par exemple, le personnel d'encadrement porte une casquette bleue. Le tableau 1 ci-dessous indique la répartition des grades avec les qualifications respectives et les niveaux de compétence, comme l'indique le Scottish Vocational Qualification ${ }^{6}$ (SVQ) ou le National Vocational Qualification ${ }^{7}$ (NVQ).

La promotion vers un grade ou niveau supérieur sera prise en compte lorsqu' un employé aura acquis le minimum de qualification requise pour un poste précis. Cette structure est relativement nouvelle. Beaucoup d'employés n'ont pas le niveau minimum de qualification requis correspondant à leur grade. En ce qui concerne les opérateurs, il existe quatre grades : A, B, C et D. Le recrutement se fait généralement

6. Qualification professionnelle écossaise.

7. Qualification professionnelle nationale. 


\section{TABLEAU 1}

\section{Structure de l'entreprise et de la qualification (les qualifications sont classées et sont validées à l'intérieur de l'entreprise, sauf avis contraire)}

\begin{tabular}{|c|c|c|}
\hline Grade & $\begin{array}{l}\text { Niveau } \\
\text { SVQ }\end{array}$ & $\begin{array}{l}\text { Qualification de base } \\
\text { pour le grade requis }\end{array}$ \\
\hline Directeurs( 5 grades)/Casquettes bleues & 6 & $\begin{array}{l}\text { Diplôme de } 3^{\mathrm{e}} \text { cycle du contrôle alimentaire }{ }^{1} \text {. } \\
\text { Cours d'hygiène (niveau du diplôme) })^{2} \text {. } \\
\text { Initiative de la Charte du management }{ }^{3} \text {. }\end{array}$ \\
\hline Contremaîtres/Casquettes jaunes & 4 & Institut de la maîtrise ${ }^{4}$. \\
\hline Chefs d'équipes/Casquettes vertes & 3 & $\begin{array}{l}\text { Certificat supérieur national }{ }^{5} \text { de la fabrication } \\
\text { alimentaire. }\end{array}$ \\
\hline O.Q./Casquettes rouges & 2 & 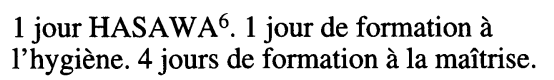 \\
\hline Opérateurs & 1 & $\begin{array}{l}\text { Stage de préparation. } \\
\text { Formation d'une durée de } 12 \text { semaines. } \\
1 \text { journée de formation à l'hygiène. }\end{array}$ \\
\hline
\end{tabular}

1. Qualification professionnelle validée par l'Institut scientifique alimentaire et de technologie, Londres.

2. Cours validé par la «Royal Society of Health».

3. Cours validé par la MCI Russel Square, London.

4. Cours validé par la ISM, Mansell House, Litchfield, Staffordshire.

5. Conseil de la qualification de l'enseignement des affaires et de l'enseignement technique.

6. HASAWA, Health and Safety at Work Act, une formation interne sur la sécurité pour répondre aux exigences de cette loi.

à partir du niveau le plus bas, c'est-à-dire, au niveau D. Un opérateur sera évalué pour être promu au grade C. Ensuite, pour accéder au grade B puis au grade A, l'opérateur devra suivre une formation spécialisée. La répartition des ouvriers entre les différents grades dépend du degré de flexibilité requis dans le service. Les travailleurs issus du grade A sont formés à toutes les opérations du service, mais pour assurer un travail efficace, il n'est pas nécessaire que tout le personnel soit dans ce grade ; la plupart des travailleurs sont aux grades B ou C. Dans un service, les opérateurs travaillent en équipe et leur travail est déterminé par leur contremaître, en fonction des exigences journalières de la production.

Pour assurer l'expansion de son plan de développement à la fin des années 1980, l'entreprise s'est posée trois questions fondamentales : «Où en sommes-nous aujourd'hui ? Où allons-nous ? Comment allons-nous faire pour en arriver là ? » Les réponses à ces trois questions impliquaient des besoins en ressources humaines. Bien que l'entreprise possédât une main-d'œuvre présentant de nombreuses compétences techniques, celles-ci ne suffisaient pas pour répondre à la vitesse de croissance des affaires et à l'accroissement de la technologie incorporée dans les investissements en capital que l'entreprise devait faire. À cette époque, la formation était organisée de façon informelle, avec peu, si tant est qu'il y en eût, de procédures permettant d'identifier les exigences en matière de formation. Peu de choses ont été faites par 
rapport à la vieille approche « sit by Nellie $e^{8}$ » (approche qui consiste à travailler en doublon avec un maître pour apprendre sur le terrain), hormis quelques formations externes ou une formation dispensée dans un collège d'enseignement supérieur de formation professionnelle. Il y avait, à cette époque, un programme de formation pour les jeunes qui était dispensé un jour par semaine dans un local technique. Ce programme a néanmoins produit des résultats médiocres, voire des normes de discipline médiocres. On n'a pas cherché à introduire des qualifications professionnelles reconnues et peu de salariés avaient une quelconque formation reconnue. Les critères de promotion étaient arbitraires et plus basés sur des relations personnelles que sur la capacité à pratiquer le métier.

L'entreprise a réussi à obtenir une subvention de l'Agence pour la formation' sous la forme d'un «Plan de formation pour la croissance ${ }^{10}$ ». Un consultant qui a été employé dans le cadre de ce contrat a persuadé l'entreprise Hall que, pour conserver un avantage concurrentiel sur ses concurrents pour la qualité des produits et la productivité, elle devait améliorer la formation et le développement du personnel. À l'issue de ce programme, un plan totalement intégré de développement des ressources humaines existantes a été accepté. Cet accord a conduit à la mise en place d'un service de formation en 1989. L'objectif de ce programme était de se doter d'une main-d'œuvre flexible avec des qualifications correspondant à leur grade (voir tableau 1).

Hall a introduit un programme de « qualité totale » selon le système de gestion de la qualité écossais appelé « Total Business Improvement Program » (Programme d'amélioration totale de l'entreprise) mettant l'accent sur la nécessité que tout le personnel s'implique dans la qualité, se développe de manière constante et améliore la qualité. L'entreprise a défini une mission et un état de la qualité. Son plan à court terme comprend un engagement de l'entreprise à travailler vers la norme « investissement en ressources humaines».

La formation est à présent étendue à tous les aspects de l'entreprise. On donne la possibilité et on encourage aussi tous les niveaux hiérarchiques de la main-d'œuvre à obtenir des qualifications appropriées. Pour ce qui concerne les opérateurs, l'accent est mis sur le développement des savoir-faire opérationnels, tandis que pour le personnel d'encadrement et la direction, on met l'accent sur le développement personnel pour permettre à cette catégorie de prendre des responsabilités au niveau de l'encadrement.

Afin de motiver les employés à suivre des formations, on les encourage à chercher à obtenir des qualifications reconnues sur le plan national comme le NVQ

\footnotetext{
8. Expression archaïque, nom d'une vieille jument âgée qui avait beaucoup d'expérience et de connaissances.

9. Training Agency

10. Business Growth Training Scheme.
} 
ou le SVQ (voir plus haut) avec davantage de formations dispensées pendant les heures de travail. Pour marquer leur réussite à un cours leur ayant permis d'obtenir une qualification, on offre aux employés de petits cadeaux comme des chopes de bière gravées.

Le NCVQ (National Council for Vocational Qualifications ou Conseil national de la qualification professionnelle) a été créé pour mettre en place des qualifications qui répondent aux exigences de l'emploi. Le but de ces qualifications est de faire partie d'une structure que tout le monde peut à la fois exploiter et comprendre. Le NCVQ est responsable de l'accréditation de ces qualifications et garantit une assurance qualité. L'objectif de ce système est de développer le personnel avec la compétence requise dans tous les secteurs pour que les entreprises puissent être compétitives sur les marchés mondiaux. Un système similaire de qualification professionnelle, le SVQ (Scottish Vocational Qualifications), a été instauré en Écosse.

Les qualifications professionnelles nationales NVQ sont obtenues sur le lieu de travail et attestent de la capacité de l'employé à exécuter dans le travail une série d'activités correspondant à des normes nationales. Chaque NVQ est composé d'un certain nombre «d'unités de compétence». Dans chaque unité, il existe une série de critères spécifiant la compétence. Les éléments sont évalués individuellement et la certification est possible dans chaque unité. Chaque NVQ reçoit un titre et un niveau qui sera placé dans une grille pour montrer comment ces qualifications sont liées les unes aux autres et comment les employés peuvent évoluer dans le système. Le principal objectif du NVQ est de créer un système de classification des qualifications cohérent et de faciliter le transfert et la progression aussi bien à l'intérieur qu'entre les unités de compétence. La grille comprend 11 zones professionnelles et cinq niveaux de compétence. Les cinq niveaux de compétence sont définis comme suit:

- niveau 1: Compétence dans la réalisation d'une série d'activités variées;

- niveau 2: Compétence dans une large gamme d'activités variées réalisées dans différents contextes. Autonomie individuelle et collaboration avec les autres;

- niveau 3: Compétence dans une large gamme d'activités variées. Haut degré de responsabilité et d'autonomie. Contrôle et orientation du personnel (conseil);

- niveau 4 : Compétence acquise dans une large gamme d'activités à la fois complexes, techniques et professionnelles. Très haut degré de responsabilité personnelle et d'autonomie. Responsabilité au regard du travail des autres et dans l'affectation/répartition des ressources ;

- niveau 5: Application d'une grande série de principes fondamentaux et de techniques complexes à travers une large variété de contextes avec 
beaucoup d'autonomie personnelle. Responsabilité pour l'analyse et dans la conception de diagnostics, planification, exécution et évaluation.

Le principal organisme du secteur est responsable des spécifications techniques pour le NVQ et définit les normes nationales. Cet organisme directeur peut être une organisation professionnelle comme un centre de formation sectoriel ou un consortium d'organismes d'une industrie particulière. Les NVQ sont évalués par un organisme agréé par le Conseil national de la qualification professionnelle qui est également responsable de l'assurance qualité et de la certification. Pour devenir un centre agréé, une entreprise (ou un centre d'enseignement supérieur de formation professionnelle, ou un organisme indépendant de formation) doit démontrer qu'elle est capable de promouvoir les compétences du personnel en conformité avec les normes nationales. Elle doit également montrer qu'elle possède les ressources lui permettant d'évaluer le personnel avec les compétences requises et démontrer qu'un système est mis en place pour assurer la qualité des futurs qualifiés. Pour des entreprises comme Hall, l'accréditation des qualifications spécifiquement orientées entraîne l'existence d'un problème de banalisation du diplôme, qui n'est pas toujours approprié, du fait de programmes conçus sur le plan national. La formation professionnelle peut à la fois mettre l'accent sur les besoins spécifiques de l'entreprise, mais elle peut aussi permettre aux stagiaires qui ont réussi leur formation d'obtenir des qualifications reconnues sur le plan national. Pour beaucoup d'employés qui ont reçu très peu de qualifications formelles au cours de leur formation à plein temps, si tant est qu'ils en ont reçues, une reconnaissance professionnelle peut être une source de fierté et de motivation et peut être utilisée pour prouver qu'ils sont capables d'apprendre davantage et d'être plus mobiles dans l'entreprise.

Le service de formation a été mis en place en 1989 et est composé actuellement d'un directeur de formation et d'un assistant ; tous deux travaillent en relation avec 20 formateurs. Chaque service comprend au moins un formateur, d'autres services en ont plusieurs; cela dépend de la taille du service. Les formateurs suivent une formation technique d'une durée de deux jours et suivent également un module Scotvec ${ }^{11}$ de formateur/évaluateur d'une durée de quatre jours. La mission principale du formateur est d'identifier et d'agréer les formations requises en collaboration avec les directeurs de services et de former ensuite les opérateurs aux compétences de base employées dans le service. La tenue de dossiers d'enregistrement des formations est un aspect important de la mission du formateur. Lorsqu'ils ne forment pas le personnel, les formateurs font des travaux de production. Par ailleurs, ils reçoivent un salaire supplémentaire pour leurs responsabilités en matière de formation, qu'ils soient amenés à former ou non. Le service de formation paie une

11. Scottish Vocational Educational Council. 
compensation au service concerné lorsque le formateur dispense une formation et qu'il ne peut, par conséquent, pas travailler au service de la production. Les programmes de développement du personnel sont directement organisés par le service de formation.

La formation est offerte et évaluée sur le lieu de travail. L'opérateur doit faire preuve de ses compétences au responsable de formation et au directeur de service, leur évaluation commune étant discutée ouvertement avec l'opérateur. La formation qualifiante suivie par un employé n'a pas de durée spécifique.

L'entreprise a été agréée comme centre de formation par le Conseil de formation aux métiers de la viande (Meat Trade Council) et comme organisme de formation par le Lothian Local Enterprise Company (LEC). En outre, l'Institut de la maîtrise a récemment reconnu Hall comme centre de formation professionnelle agréé habilité à donner des cours de maîtrise et de responsables pour cet organisme accréditeur. On encourage le personnel d'exécution à obtenir des qualifications professionnelles reconnues sur le plan national et transférables comme les NVQ et SVQ.

Le service de formation de Hall est devenu une partie intégrante de l'usine avec la mise en place de programmes de formation développés dans la plupart des secteurs de l'entreprise. L'approche générale est très centrée sur les formations reconnues nationalement, par le biais de la formation sur le tas, avec une évaluation des compétences sur le lieu de travail. Quelques-unes des difficultés éprouvées lors de l'utilisation d'organismes de formation, et plus précisément au moment où les chefs d'équipe étaient parfois réticents à autoriser des formateurs à former, ont été résolues par la création d'un nouveau poste de responsable de formation qui dépend directement du directeur de la formation. La responsabilité de la rémunération des formateurs et leur gestion ont été transférées au service de formation. Cette séparation rend les coûts bien plus transparents, ce qui pourrait avoir des conséquences positives sur l'efficacité et sur les coûts de la formation. Les responsables de la formation sont formés et évalués. L'objectif est de promouvoir les compétences de l'ensemble du personnel, puisque c'est un moyen qui permet d'améliorer l'efficacité au sein de l'entreprise et qui contribue à réduire le gaspillage, d'où l'amélioration des performances de l'entreprise. Des relations verticales existent également sur ce plan entre l'entreprise Hall avec ses principaux fournisseurs, les agriculteurs, et ses principaux clients (les détaillants).

\subsection{Unibon (Italie)}

Unibon est une société coopérative à responsabilité limitée située dans la région de Modena dont l'activité est centrée sur la transformation des viandes porcines et bovines. Les associés de cette coopérative sont des éleveurs (1 300 environ). Unibon a été créée précisément le $1^{\text {er }}$ janvier 1991 à partir de la fusion de deux sociétés coopératives : CIAM située à Modena et ACM située à Reggio Emilia. Ces deux 
coopératives ont été lancées après la Seconde Guerre mondiale au sein d'un mouvement coopératif italien plus vaste, Lega Nazionale delle Cooperative e Mutue. Elles sont implantées dans une région très développée de l'Italie, l'Émilie-Romagne, où la forme coopérative est particulièrement répandue ${ }^{12}$. Depuis la création d'Unibon, des rachats ont été effectués dans le même secteur.

Le Groupe Unibon consolidé représente, en 1994,

- trois divisions : Italcarni (abattage des porcs), Unicarni (abattage des bovins), Unibon (charcuterie industrielle);

- 10 établissements ( $120000 \mathrm{~m}^{2}$ de surface construite);

- 1077 salariés et 2625 associés ;

- 100000 bovins et 350000 porcs abattus par an.

En 1993, le chiffre d'affaires consolidé s'élevait à 262 millions d'ÉCU. Pour Unibon, il était de 113 millions d'ÉCU.

Nous centrerons notre attention sur Unibon SCRL et ses deux établissements de Modena et de Reggio Emilia. La fusion de ACM et CIAM a permis d'obtenir des gains de productivité importants et a conduit à une réduction progressive des effectifs (de 607 en 1990 à 547 salariés en 1994). La rationalisation opérée lors de la fusion a entraîné une certaine spécialisation des établissements. À Modena sont produits les divers saucissons et salami, la mortadelle, les jambons cuits, tranchés et emballés sous vide. À Reggio Emilia sont fabriqués les différentes variétés de lard et de bacon, les viandes industrielles, les jambons cuits, emballés ou précuits. Le processus de production comprend, à partir de l'abattage des animaux provenant des éleveurs associés, toutes les étapes de découpe, de préparation et de cuisson propres à la charcuterie traditionnelle. Selon les produits et la qualité finale voulue pour le marché, les étapes sont plus ou moins automatisées. Ainsi pour les jambons, après injection de la saumure, l'entreprise est-elle une des rares à disposer d'une machine qui réalise le massage nécessaire à la diffusion de la préparation dans l'ensemble de la cuisse. Pour les deux usines, on trouve également six lignes de conditionnement automatisées permettant de fournir des marchés étrangers difficiles sous un emballage satisfaisant (USA, par exemple).

Le marché principal de l'entreprise est l'Italie. Cependant, près de $20 \%$ des produits sont exportés en Europe, mais aussi aux États-Unis. Ce dernier pays a d'ailleurs nécessité depuis longtemps un effort important de l'entreprise en matière de qualité et d'hygiène pour répondre aux exigences américaines concernant l'importation des produits alimentaires. Le marché est très concurrentiel et cette

12. C'est une région tout à fait typique de l'organisation spatiale industrielle sous forme de districts industriels ( $c f$. Bagnasco et Sabel, 1994, par exemple). 
concurrence est très fragmentée. Tout en faisant partie des 50 plus grandes entreprises de l'agroalimentaire italien et en étant en première position pour les jambons de Parme, en cinquième pour la charcuterie ou en troisième pour les jambons cuits, les parts de marché de Unibon sont de l'ordre de 3 à $5 \%$ par rapport aux autres sociétés italiennes. La stratégie progressive consistant à réaliser des fusions ou des acquisitions d'entreprises régionales similaires visant ainsi à faire face de manière plus assurée à cette fragmentation. La compétition sur les prix, à la fois en amont (prix du porc ${ }^{13}$ ) et en aval pour les produits diffusés, a d'ailleurs fait perdre près de 2 millions d'ÉCU à l'entreprise en 1993, malgré une progression globale des ventes. Les ventes de charcuterie à l'étranger (principalement en Allemagne et en France) mais aussi en Angleterre, en Grèce, en Équateur, etc.) sont en forte croissance (augmentation de $50 \%$ entre 1992 et 1993); cependant, elles ne représentent que quelques points de pourcentage du chiffre d'affaires.

Depuis 1988, une politique de «qualité totale» d'envergure a été mise en œuvre dans l'ensemble des aires de l'entreprise. La rationalisation nécessaire des activités pour espérer progresser face aux concurrents a conduit la direction de l'entreprise à adopter les techniques de TQM en faisant particulièrement appel à un consultant américain d'origine japonaise : le professeur Shoji Shiba qui continue à visiter régulièrement l'entreprise une ou deux fois par an. Cette politique n'est pas seulement une vue de l'esprit ou un discours de dirigeant. À plusieurs niveaux dans l'entreprise, les gens ont «joué le jeu». Concrètement, une cellule de deux personnes a été créée pour animer le TQM au quotidien. Elle est rattachée à la direction de l'organisation et du personnel. Le responsable est un ancien cadre de Fiat (passé dans les fonctions marketing et organisation) qui est assisté par un technicien. Ce programme global d'amélioration des pratiques de l'entreprise utilise principalement une technique de résolution de problèmes qui a été enseignée sur des cas réels et qui a été largement diffusée auprès des salariés. Les rôles de la cellule TQM sont d'aider les groupes à se mettre en place, de leur fournir des techniques d'analyses adaptées au problème, puis de piloter de manière plus distante l'avancement du groupe en étant particulièrement attentif à la motivation de ses membres. La cellule gère également certains aspects de la formalisation des productions des groupes de travail (plaquettes, procédures écrites, bilan des activités des groupes, etc.).

On peut dire que cette activité TQM s'appuie sur une tradition ancienne et locale de coopération entre les salariés et la direction. Certains éléments du TQM ont fait l'objet d'une négociation avec les représentants syndicaux et sont intégrés dans les accords d'entreprise. Par exemple, les réunions des groupes doivent avoir lieu pendant le temps de travail, des formations d'accompagnement des membres

13. Pour donner un ordre de grandeur : le prix du porc au kilo a baissé de $16 \%$ entre 1992 et 1993. 
des groupes de travail sont prévues; les bénéfices économiques éventuels des améliorations peuvent être intégrés dans le salaire variable.

De même, un comité paritaire de gestion de la qualité discute des thèmes d'amélioration choisis, de la composition des groupes de travail et assure un suivi de l'avancement et des résultats des travaux.

De 1988 à 1994, le service TQM a calculé la répartition des heures de travail effectuées dans ces opérations : 3500 heures de formation, 6600 heures de travail des groupes, 2100 heures pour la diffusion des résultats, 1200 heures pour la coordination des groupes, 840 heures pour les opérations BRAVO ${ }^{14}, 640$ heures de visites des clients, 300 heures pour les concours d'innovations « Archimède». Sur 44 groupes actifs dans cette période, 11 étaient des groupes réunissant plusieurs fonctions de l'entreprise et la grande majorité regroupait des salariés de plusieurs niveaux hiérarchiques. La répartition des problèmes traités par les groupes était la suivante : 20 en production, 10 pour le commercial, cinq dans l'administration, cinq relatifs aux accidents du travail et à l'hygiène et quatre pour les services généraux. Quelques exemples de problèmes traités par les groupes : réduction des défauts de distribution de sel et de lard dans la mortadelle, amélioration de la sélection des jambons de Parme, réduction du nombre de produits indisponibles à la livraison, réduction des retours de produits de la part des clients, définition des procédures pour le respect des normes d'hygiène, réduction des accidents de travail dus aux moyens de transport internes, etc.

Voici quelques témoignages de salariés qui montrent le type de travail réalisé dans ces groupes:

BRAVO c'est pour l'ordre et la propreté, on s'arrête pendant 4 heures un jour de printemps, l'usine aussi, et tout le monde nettoie et range dans son coin de travail (employé).

\section{V. directeur du personnel souligne :}

C'est devenue une technique de management généralisée, ç'a influencé notre style. Par exemple, quand il y a une erreur, plutôt que de rechercher le coupable uniquement, il faut repérer les causes et les solutions, donc: TQM [...] Le TQM s'occupe des problèmes stratégiques, et la direction est dans les groupes de travail. De 1988 à 1991, c'étaient des thèmes faisables facilement pour former l'entreprise, pour éviter les frustrations et les désillusions. Au fur et à mesure, les thèmes deviennent stratégiques. C'est le cas des sept groupes qui travaillent sur les produits fondamentaux de Unibon. Ces groupes amènent à la direction, à partir de l'analyse de la concurrence, des propositions de choix stratégiques [...] Le président, c'est lui qui a introduit tout ça, dans le comité de direction, l'approche, est vraiment TQM.

14. BRAVO : Buttare, Riordinare, Agevolare, Verificare, Osservare, c'est-à-dire « Jeter, Classer, Améliorer, Vérifier, Observer (les règles) ». 
Une participante à l'opération «A tutto cotto $^{15}$ » raconte :

On avait perdu $10 \%$ du marché en 1993 sur les produits cuits alors que ça représente $30 \%$ du chiffre d'affaires. En trois mois, on a fait quatre groupes : sur les consommateurs, les clients, les leaders d'opinion et la concurrence. Ç'a marché, car on est remonté de $8 \%$. Mais j'étais dans le groupe sur les leaders d'opinions. On a fait du $\mathrm{TQM}$, mais de manière très rapide, très focalisée. On a fait des questionnaires, des entretiens [...] Pour les leaders, on se demandait : qui sont-ils ? Comment influencentils ? C'était assez abstrait, théorique, pas très « TQM». Le groupe comprenait un viceprésident, un président d'une autre société du Groupe, le directeur de production et un consultant en relations publiques. On a identifié les médecins, les nutritionnistes, les diététiciens, les médecins du sport, des gens qui travaillent à l'université, qui sont consultants pour la restauration collective, certains journaux... Les médecins généralistes ont peu de poids là-dessus, c'est différent pour les pédiatres. On a eu des contacts directs, on s'est rendu compte que les nutritionnistes connaissaient bien les jambons, mais qu'ils sont pour la plupart végétariens ! Les pédiatres, les hôpitaux recommandent le jambon cru mais sans savoir pourquoi ! Ça nous a amenés à revoir nos étiquetages, le packaging, on leur a dit qu'on mettait nos moyens, notre savoir à leur service, c'était très intéressant pour eux aussi [...] Un exemple : les pédiatres qui avaient des bébés allergiques au lait disaient que le jambon c'était pas bien, car ils croyaient que le jambon se cuit dans du lait, donc ça veut dire qu'il faut aussi mettre la technologie de cuisson sur les étiquettes.

Un cadre présente un bilan de son expérience :

On met en commun nos connaissances, on voit les problèmes communs. Le deuxième intérêt, c'est la motivation, on voit les résultats sauf sur les projets très longs, c'est frustrant, par exemple pour les normes, le processus est très long. L'année prochaine, on aura «A tutto Salame » en plus de «A tutto cotto».

Pour le responsable de la cellule TQM, le mouvement paraît bien lancé ; beaucoup de personnes se sont appropriées les méthodes. Cependant, l'accent est mis sur un état d'esprit et des pratiques quotidiennes : «On a voulu séparer le TQM des problèmes de qualification et de la course à la carrière. L'objectif reste d'améliorer l'ensemble de l'entreprise même si le TQM peut servir parfois de révélateur pour les individus et permet de les repérer pour leur carrière . »

L'entreprise comptabilise assez précisément l'effort de formation effectué. C'est également la cellule TQM qui suit l'activité formatrice. Malgré l'absence de contraintes légales en Italie, on peut dire que la formation joue un rôle important bien que variable selon les niveaux hiérarchiques ou les fonctions.

Comme nous l'avons souligné, la présence syndicale est importante dans cette entreprise, à la fois par rapport à la forme coopérative de cette société de l'agroalimentaire et par rapport au milieu local de l'Émilie-Romagne. Pour autant,

15. Groupe de travail sur le jambon cuit (jeu de mots avec l'expression «c'est tout cuit »). 
entre les établissements de Reggio Emilia et de Modena, des différences importantes apparaissent entre les délégués malgré l'appartenance commune de certains au même syndicat CGIL.

\section{Pour les représentants de l'établissement de Modena (CGIL et RSU)}

[...] les présentations du professeur Shiba nous rendaient sceptiques. Importer une philosophie japonaise, c'était pas évident. Ici, les rapports sont bons avec la direction, ils n'ont pas d'à priori contre les syndicats. Par contre, la province (au niveau syndical) n'est pas d'accord avec le TQM sur le plan idéologique. La participation des travailleurs à l'intérieur des coopératives est bien sûr favorisée, mais avec le TQM, le problème, c'est qu'il n'y a pas tellement d'implications sur les rémunérations. On pensait aussi que ça affaiblirait les syndicats mais nous, après quelques années, on ne peut pas dire que ce soit le cas. Il y a toujours des thèmes de conflit. [...] Ici, il y avait de l'enthousiasme au départ quand on était encore CIAM. On a pu traiter des petits problèmes. On a eu des résultats très concrets. Il y a même eu une fête après la signature d'accords intégrant le TQM. Mais l'unification de 1991 a un peu cassé l'enthousiasme. Le TQM a été plus utilisé à d'autres endroits que pour la production. Ç'a évolué vers les problèmes de marché, de gammes de produits. Finalement, ç'a donné un peu l'impression que tout le travail fait, c'était de la publicité pour l'extérieur. [...] Concrètement, il y a toujours des volontaires ; les travailleurs ont plein de solutions dans leur tête pour diminuer la fatigue, augmenter la sécurité. Il suffit simplement de réfléchir. [...] Il y a un comité de gestion de la qualité avec le président, les leaders des groupes et les représentants syndicaux du conseil d'entreprise. Il choisit les problèmes et propose des noms pour y travailler : $99 \%$ des gens acceptent. Maintenant, le niveau des problèmes a augmenté et les ouvriers sont de moins en moins consultés. La grosse critique, c'est que tout ça permet seulement d'améliorer la productivité. Le TQM, c'est un problème d'entreprise et pas de travailleur. Pourquoi arrêter au niveau ouvrier, il y a de nouveaux problèmes qui surgissent. C'est vrai qu'on a diminué les accidents du travail ; ç'a permis de mieux connaître ce qui se passe dans l'entreprise en augmentant l'esprit d'équipe. Mais financièrement, pour les travailleurs, ça n'a pas été vraiment ce qui était prévu. Le problème aussi, c'est que nos efforts de qualité n'empêchent pas que le problème principal, c'est la matière première, les fournisseurs.

Du côté de Reggio Emilia, la principale responsable syndicale (CGIL) tient un discours moins nuancé :

Avant la fusion, on avait une co-détermination sur beaucoup de choses ; on discutait l'influence de la stratégie. Il y a eu 300 à 400 pages d'accords signés. On trouvait un accord sur tout ! L'idée, c'était «gouverner en recherchant l'accord des salariés». Depuis 1991, les relations sociales se sont modifiées, les problèmes de fusion, de stratégie ont fait passer les conditions des travailleurs au second plan. Ils ne recherchent plus le consensus. [...] On a renouvelé en partie les accords en août 1994. Sur certains points, on a essayé de rapprocher les deux établissements, mais il y a encore beaucoup de choses. Nous, on était plus en avance pour les relations avec les syndicats. Par exemple, en 1988, on avait mis en place les «aires intégrées », on essayait de faire participer les travailleurs à la gestion, aux décisions qui avaient des effets sur le travail 
dans chaque aire. Il y avait une réunion par mois, tous pouvaient participer. [...] En face de ça, le TQM, les KIT, les groupes, ça exclut la collectivité, car le groupe ne peut pas être important. Ça exclut les discussions collectives, ça individualise les problèmes.

[...] Ce qui me choque, c'est qu' on vende cela comme de la «participation », mais c'est de l'illusion. Maintenant, le seul truc qui nous reste, c'est une réunion par semaine sur le programme travail. Avant, on avait une « commission bilatérale de projet » avec les délégués et des experts. On apprenait, on discutait des investissements. Il fallait l'accord des deux parties. C'était une vraie participation.

[...] Le problème aussi, c'est que les groupes de base qualité annulent le rôle du syndicat. Mais nous, on reste comme des syndicalistes dans les groupes de base. Mon rôle est d'influencer, de tenir compte de la plate-forme nationale du syndicat. [...] Comparativement à Modena, nous, nous avons maintenu des choses; eux, ils en ont vraiment acquis de nouvelles. [...] Le salaire variable, on a signé, mais on a de gros doutes sur la capacité des gens à comprendre ce qui le fait varier. Il faut savoir comprendre un bilan, savoir que les amortissements, on peut jouer dessus. [...] Dans ma conception, la participation, c'est pas seulement l'efficience de la production, même si je suis tout à fait pour améliorer l'efficience : par exemple, on ne devait pas avoir de groupe de travail sur les rendus. Pour moi, c'est un manque de participation qui fait apparaitre ce problème. Un travailleur n'aurait pas laissé passer un certain nombre de trucs s'il y avait eu une vraie participation.

\subsection{ABC Ltd (Irlande)}

Fournisseur international de pizzas congelées, de poissons et de pâtisseries salées, ABC Ltd approvisionne aussi bien des distributeurs sous la marque du fabricant que des distributeurs qui ont leurs propres marques. Depuis son démarrage en 1981, ABC Ltd est devenue l'une des plus grandes réussites dans le secteur agroalimentaire irlandais. L'entreprise emploie actuellement plus de 800 personnes en Irlande. En 1994, son chiffre d'affaires dépasse les 100 millions de livres sterling. Depuis sa création, le groupe n'a cessé de progresser grâce à sa stratégie d'innovation des produits, d'investissement technologique et de développement de la marque. L'entreprise est leader sur le marché du poisson et de la pizza et a lancé des marques à succès dans les deux secteurs.

La maison mère, qui est implantée dans le comté de Dublin, a été créée en 1981. Le fondateur de l'entreprise travaillait auparavant pour une des plus grandes chaînes de distribution en Irlande. Alors qu'il travaillait en tant qu'acheteur, il identifia un marché d'approvisionnement non exploité, celui de la fabrication et de la distribution de pizzas fraîches et congelées en Irlande. En collaboration avec le cofondateur de l'entreprise, il a investi plus de un million de livres sterling dans la création de l'entreprise. De plus, ABC Ltd a bénéficié d'une subvention de l'Agence irlandaise du développement (Irish Development Agency que nous appellerons IDA). 
Dans un premier temps, le fondateur de l'entreprise ABC Ltd a soumis une proposition à l'IDA en 1982. Son intérêt se portait sur les fonds disponibles pour la recherche et le développement dans le secteur de la pizza. Dès le départ, l'IDA ne considérait pas le fondateur d'ABC Ltd comme un entrepreneur banal. Il était vu comme un homme à la fois dynamique et ayant un sens aigu des affaires. Ainsi, on l'a immédiatement placé dans le programme de développement d'entreprise (Enterprise Development Programme : EDP).

En Irlande, le groupe $\mathrm{ABC}$ Ltd exploite cinq usines de transformation alimentaire :

- deux usines de fabrication de pizzas,

- une usine de transformation des poissons,

- une usine de fabrication de pâtisseries salées (qui fait l'objet de cette étude).

Dans ce texte, une attention particulière sera portée à l'usine de Galway ${ }^{16}$. Ses efforts sont centrés sur la production de tourtes salées, de feuilletés de saucisses et de crêpes salées. Galway a été choisie, car le taux de chômage élevé dans cette région signifie qu'il y a une importante disponibilité de la main-d'œuvre. Après avoir démarré son activité avec 70 personnes dans l'usine, ABC Ltd compte actuellement un total de 280 employés et continue son expansion.

La période de démarrage au sein de l'usine fut caractérisée par un comportement général en recherche et développement qualifié de «chaotique»par le directeur de la production. $\mathrm{ABC}$ Ltd s'est livrée à une lutte sans merci pour trouver un produit lui permettant d'assurer sa survie et, en définitive, une rentabilité significative. Les tourtes ont été choisies, car elles semblaient à même de convenir à cette description. Au départ, l'entreprise a essayé de produire toutes sortes de tourtes, y compris les tourtes sucrées, les tartes aux pommes, les tourtes «Bakewell» et les tourtes salées. Le marché des tourtes salées s'est révélé moins concurrentiel. L'entreprise s'est centrée sur elle. Cependant, les limites du marché irlandais pour les pâtisseries salées ont rapidement été identifiées. En effet, on a constaté que le marché irlandais dans sa totalité pouvait être approvisionné par la production d'un après-midi. L'entreprise se trouvait en face d'un dilemme de nature technologique : la meilleure efficacité technologique centrée sur les coûts conduisait immédiatement à une saturation du marché national.

La fusion terminée, la fabrication de quiches a remporté un vif succès jusqu'au jour où une ministre britannique, Edwina Curry, a souligné au Parlement britannique les problèmes d'empoisonnement par la salmonelle dans les œufs. Les conséquences

16. Galway : troisième ville de l'Irlande. 
ont été dramatiques : la demande en quiches a immédiatement chuté de 600 à deux caisses par semaine et on a dû, en définitive, abandonner la production. La perte de l'entreprise a été considérable, aussi bien en termes d'investissement qu'en matière de capital, de temps gaspillé et de savoir-faire acquis.

À travers son actionnaire Northern Foods, $\mathrm{ABC}$ Ltd a pu répondre à l'appel d'offre de l'entreprise Birds Eye ${ }^{17}$ au Royaume-Uni. Cette offre a abouti à une commande en «co-emballage» de la part de Birds Eye.

En 1989, la production de tourtes a pratiquement doublé par rapport à l'année précédente, ce qui a permis à l'entreprise d'exploiter une seconde chaîne de production. Dans la même année, l'entreprise a acquis une chaîne de production de pâte feuilletée japonaise qu'elle a utilisée pour la production de feuilletés de saucisse.

Au début de l'année 1990, ABC Ltd a décidé de se lancer dans le marché des pâtisseries salées. La production a été localisée dans l'usine de Galway. Le changement orienté vers la production de crêpes a été possible, sans pour autant faire l'acquisition d'une nouvelle usine. L'objectif était de produire une pâte à frire qui pouvait être couverte par une sauce salée. La récente ligne de pâte feuilletée japonaise a été utilisée à partir de l'expérience de la fabrication sur commande des feuilletés de saucisse. L'équipe a décidé de «modifier légèrement » la technologie existante pour mettre au point une enveloppe de crêpe. L'équipe de l'usine travaillait sur la ligne de pâte de feuilleté japonaise existante et a réussi à produire une enveloppe de pâte à frire panée qui pourrait être couverte et garnie en utilisant la ligne de pâtisserie.

Dans sa troisième forme, l'entreprise $\mathrm{ABC}$ Ltd se considère comme préparatrice en aliments salés, réduisant ainsi de manière soutenue les activités qui nécessitent une forte main-d'œuvre aux dépens de leurs fournisseurs de matières premières. $A B C$ Ltd a insisté sur le fait que les fournisseurs doivent s'orienter vers la pré-préparation des ingrédients, c'est-à-dire le nettoyage, le décorticage, le découpage en tranches et l'épluchage des produits «matières premières ».

La technologie employée par l'entreprise $\mathrm{ABC}$ Ltd est la plus récente et a toujours constitué le moteur du succès de l'entreprise. Conformément à leurs comportements en matière d'innovation, la direction et le personnel ont fréquemment travaillé en étroite collaboration pour améliorer la technologie existante. Cela est connu dans l'entreprise comme «le fait de faire des petites modifications », ce qui peut conduire jusqu'à une reconception d'une ligne de production entière. Les modifications sont réalisées par une petite équipe de l'usine composée de deux opérateurs en ligne, d'un ingénieur et d'une équipe technique. Les dépenses en investissements pour de nouvelles machines sont élevées. Le directeur d'usine est

17. Birds Eye : marque de produits congelés. 
extrêmement fier de la façon dont toute l'équipe de l'usine a pu introduire de nouveaux produits en exploitant des lignes de production destinées initialement à la fabrication d'autres produits. Il cite l'exemple de la production de crêpes sur une chaîne d'abord destinée à la fabrication de la pâtisserie. Les modifications technologiques pour améliorer la rentabilité sont constantes, ce qui conduit à de fréquents développements sur les lignes posant sans cesse le problème d'identifier les obstacles et de trouver des moyens pour les surmonter.

Les chaînes de production sont conçues pour être aussi conviviales que possible, de telle sorte que les opérateurs, avant d'être opérationnels sur les chaînes de production, n'aient besoin que de peu de formation. La salle de formation multimédia est située au même étage que celui de la production. Dans cette salle, les employés ont la possibilité de suivre des «formations assistées par ordinateurs ».

Le p.-d. g est très impliqué dans un processus d'information et de prise de décision ouverte. Mensuellement, des réunions de groupes de décision centrés sur les problèmes ont lieu. Les participants à ces réunions, à tous les niveaux de l'entreprise, signalent les points que l'on doit améliorer, chaque participant repart avec du travail à faire. Ils présentent également le bilan de ce travail au cours de la réunion suivante. Le directeur de l'usine perçoit le résultat de telles réunions comme un moyen permettant à l'équipe de l'usine de combiner ses savoir-faire et ses connaissances et de parvenir à une vision commune. Il conçoit les groupes de travail comme un élément critique pour permettre le maintien d'un bon moral et d'une forte créativité à tous les niveaux de l'usine. Le directeur de l'usine souligne que le personnel jeune a réagi très positivement à ces prises de responsabilité rapides. Comme il le souligne, cela conduit les employés à prendre des responsabilités personnelles et à se sentir responsable. Il encourage une totale liberté d'expression lors des réunions, en allant jusqu'à proposer lui-même des suggestions stupides, cela pour démontrer aux jeunes issus des équipes que même les grands managers ne sont pas omniscients.

$\mathrm{ABC}$ Ltd est la principale industrie de la ville. La majorité des employés est issue d'un milieu rural. Le chômage peut atteindre $30 \%$ dans la localité (ce taux est particulièrement élevé par rapport à la moyenne irlandaise) et le principal choix s'offrant aux jeunes qui ont quitté l'école est l'émigration. Les États-Unis ou le Royaume-Uni sont les destinations favorites. Dans le passé, beaucoup d'employés sont partis aux États-Unis, mais ont dû retourner en Irlande plus tard, à cause des moindres possibilités d'emploi. Environ $90 \%$ des employés sont issus des écoles locales. Pour les opérateurs, le niveau d'études exigé est le certificat de fin d'études. Habituellement, les nouveaux employés ont une certaine expérience du travail à l'usine à temps partiel. Lors du démarrage de ses activités, l'usine avait pour politique de recruter les jeunes ayant quitté l'école et qui étaient sans emploi. Selon le directeur général, deux raisons expliquent cette décision : d'un côté, l'entreprise a cherché à s'intégrer au sein de la communauté ; de l'autre, ce recrutement était un 
moyen d'atténuer le problème croissant du chômage chez les jeunes. L'usine est une communauté très soudée, tout le monde entretient des relations avec autrui, en dehors du travail. Souvent deux ou trois personnes provenant de la même famille sont employées dans l'entreprise. Au sein de l'usine, il est habituel de voir des parents ou des amis proches qui travaillent en tant que formateurs ou conseillers des nouveaux employés.

Lorsque $\mathrm{ABC}$ Ltd a été créée, la formation n'était pas considérée comme une priorité et elle était très informelle. Au départ, $A B C$ Ltd a bénéficié de subventions de la FAS, dans le cadre du Plan d'aide à la formation (Training Support Scheme), et de l'IDA, à travers un programme sous le «New Industry Training Grants Scheme». La FAS est un organisme à la fois public et privé qui s'occupe de la formation en Irlande; un de ses principaux rôles est de promouvoir la formation en finançant de manière sélective les formations. L'IDA est la seconde agence publique irlandaise qui s'occupe de la recherche et du financement des formations pour les entreprises et de programmes de développement dans l'industrie. ABC Ltd a disposé de subventions de l'IDA (en collaboration avec la FAS).

Cependant, ces subventions n'ont pas permis de mettre en place des politiques structurées de formation. Les principaux catalyseurs ayant permis la mise en œuvre d'une stratégie formelle de formation émanaient de trois éléments bien distincts : l'introduction des normes ISO 9000, la demande de la clientèle et la demande des employés.

Dans le cadre de l'ISO 9000, un dispositif de formation a été mis en place. $\mathrm{ABC} L \mathrm{Ltd}$ a dû tenir des dossiers de formation et formuler des objectifs de formation précis pour l'entreprise.

Les exigences des clients entraînent souvent un certain niveau de formation dans l'entreprise. Par exemple, Sainsburys a introduit le contrôle des dossiers de formation comme un des critères d'évaluation de la qualité des produits.

Une étude conduite au sein de l'entreprise a révélé que les employés attendaient plus que des encouragements financiers : ils attendent également reconnaissance et développement de leurs compétences. Cela a été pris en compte dans la politique de ressources humaines qui continue de mettre l'accent sur l'accroissement de la formation et sur des méthodes plus conviviales d'apprentissage. Dans une moindre mesure, la direction a le sentiment que les lois ont aussi été un élément catalyseur pour la mise en place de la formation, en particulier la législation sur la santé, la sécurité, l'hygiène et la qualité. À partir de 1995, une exigence en matière de formation fait partie intégrante du contrat de travail ; elle est liée à la performance, à la rémunération et à la promotion.

Lorsqu'un employé nouvellement embauché commence au sein de l'entreprise, on procède à l'ouverture d'un dossier de formation. Dans ce dossier figure 
le nom de l'employé, le titre de l'emploi, la date à laquelle l'employé(e) démarre, la nature et le contenu des formations ainsi que leur durée. Dans ces dossiers sont indiquées toutes les formations suivies par les employés, aussi bien internes qu'externes à l'entreprise. Le premier stage qu' un nouvel employé doit suivre est le séminaire d'intégration. Au cours de ce séminaire, on présente le groupe $\mathrm{ABC}$ Ltd ainsi que la gamme des produits de l'entreprise ; ce séminaire d'intégration permet aux nouveaux de mieux se situer au sein de l'entreprise.

Un plan général de formation et de développement est élaboré et révisé sur une base annuelle par le directeur général de l'entreprise. On le décline ensuite dans un plan de formation interne pour chaque secteur au sein de laquelle les opérateurs sont employés : emballage, expédition, production, etc. Chaque plan de formation de chaque département est composé de modules spécifiques. Tous les modules du plan de formation doivent être suivis au cours de la période d'essai de l'employé. Le responsable de formation ne signera pas la feuille de formation tant qu'il estime que la personne en formation n'est pas pleinement compétente pour sa section spécifique.

Au départ, la formation était très informelle et les employés les plus anciens de l'entreprise devenaient formateurs. Comme la structure de l'entreprise s'est développée, les dispositifs de formation se sont également organisés. La formation est devenue davantage un choix de l'employé : on lui demande en effet de plus en plus son avis sur le type de formation à suivre.

Dans une salle de formation adjacente à l'atelier, l'employé peut bénéficier d'un enseignement assisté par ordinateur. On encourage chaque employé à apprendre seul et selon son propre rythme. Le retour d'informations sur ces modules révèlent que les stagiaires sont satisfaits et que la formation répond bien à leur besoin. À l'issue de la formation, chaque employé reçoit un certificat reconnu dans l'ensemble du Royaume-Uni.

Bien que la plupart des formations soient dispensées à l'intérieur de l'entreprise, on fait occasionnellement appel à des consultants privés. Les employés ont aussi la possibilité de participer à d'autres formations s'ils le souhaitent. Un pourcentage des frais de ces stages est pris en charge par l'entreprise jusqu'à une limite de 500 livres sterling par an. Jusqu'ici, comme cela n'a pas été divulgué aux employés, seulement trois ou quatre personnes ont pu en profiter.

\section{Conclusion}

Face à la diversité des pratiques, il parait utile de fournir quelques points de repères. En premier lieu, les actions de formation se situent par rapport à deux pôles, du plus simple au plus complexe. Pendant longtemps, les actions de formation avaient un point d'application limité. Il s'agissait, par exemple, de former un groupe 
d'ouvriers à acquérir certaines pratiques d'hygiène. En revanche, on observe de plus en plus que, pour des raisons d'efficacité et en raison de leur rôle stratégique, ces activités sont intégrées au fonctionnement de l'entreprise, qu'il s' agisse de l'exercice du travail lui-même, d'une démarche d'amélioration permanente des processus de travail ou des systèmes de qualification et de classification des emplois au sein de l'entreprise. Un second point concerne l'organisation des actions de formation. Traditionnellement organisées de manière systématique pour un groupe de personnes, elles prennent de plus en plus la forme d'actions plus individualisées basées sur l'auto-apprentissage. La durée et le rythme d'apprentissage sont de plus en plus adaptés aux caractéristiques et aux contraintes individuelles. Un troisième élément concerne le degré de formalisation des actions de formation dispensées. S'agit-il, par exemple, d'un enseignement organisé, ou davantage d'une formation plus informelle intégrée dans les pratiques de travail quotidiennes? Une dernière grande différence concerne le cadre de référence de l'action de formation. Longtemps considérées comme des activités devant permettre aux individus de mieux s'adapter aux évolutions des procédés de fabrication, les actions de formation sont désormais souvent employées comme un levier d'adaptation continue de l'ensemble de l'entreprise aux changements de marché et de stratégie.

Les entreprises présentées diffèrent par leur taille et partiellement par la nature de leurs activités. Plusieurs points communs les rapprochent cependant : l'intensité de la concurrence, la recherche de la qualité des produits, la préoccupation de l'hygiène, la recherche de la valeur ajoutée (en particulier, pour éviter la concurrence des pays d'Europe centrale dont les prix sont très concurrentiels sur des produits à faible valeur ajoutée).

Les comportements les plus innovants observés en matière de formation continue dans les cas étudiés présentent ainsi les caractéristiques suivantes :

- le rôle formateur de l'encadrement est particulièrement déterminant. Ce rôle peut être apparenté à celui d'un « traducteur » au sens où Michel Serres ${ }^{18}$ utilise ce terme. Le recours à l'encadrement permet de résoudre plusieurs difficultés particulièrement prégnantes en PME : adaptation des connaissances transmises au contexte pratique dans lequel évolue les salariés, ce qui rend l'assimilation et l'utilisation de celles-ci beaucoup plus faciles ; pas d'arrêt du processus de production comme peut le provoquer l'envoi de salariés en stage à l'extérieur, prise en compte du niveau de compétence réel des salariés, ce qui est un atout pédagogique évident. Les différents cas étudiés montrent que cet aspect dépend très peu du contexte et peut donc être facilement transféré. Paradoxalement, la situation française peut constituer un obstacle sur ce plan, car le système d'encouragement à

18. Voir Michel Serres (1992), par exemple. 
la formation ${ }^{19} \mathrm{a}$ du mal à reconnaître ce genre de pratiques insuffisamment formalisées ;

- l'intégration forte entre la politique de formation et la stratégie de l'entreprise, l'une et l'autre se soutenant de manière cohérente, est apparue comme une source de succès dans l'évolution positive conjointe de la professionnalité des salariés et de la croissance économique de l'entreprise. Certaines entreprises sont en quelque sorte obligées de fonctionner ainsi lorsque le niveau professionnel des salariés est faible ou peu adapté au secteur d'activités (Luxfood et Hall, par exemple). Mais on constate également avec le cas de Unibon qu'il peut s'agir d'un processus permanent. Pour transférer ce genre de pratiques, on relève que la formation et, en particulier, le service qui la pilote ne doivent pas être isolés comme une activité fonctionnelle d'appui, mais être intégrés au fonctionnement quotidien de l'entreprise, depuis la production de base jusqu'au comité de direction ;

- les pratiques de formation intégrées participent ainsi de l'évolution à «petits pas» de l'entreprise. Nous n'avons pas observé de « révolution technologique » dans ces cas ; plutôt des adaptations progressives des salariés aux machines, mais aussi des machines aux capacités des salariés qui, formés suffisamment, sont capables de proposer des adaptations techniques permettant certaines évolutions stratégiques de l'entreprise. Les équipes de reconception chez Hall ou les groupes de résolution de problèmes chez Unibon illustrent particulièrement cette évolution réciproque et progrédiente des hommes et des techniques;

- un élément important de ces activités de formation concerne l'enregistrement, la mémorisation des expériences des salariés. Un suivi très détaillé permet en effet de gérer de manière fine la progression des salariés et l'évolution des réponses à apporter en matière de formation. La situation au RoyaumeUni apparaît, cependant, beaucoup plus en avance sur ce plan que dans les autres pays puisque cette gestion des qualifications n'a pas seulement un usage interne, mais constitue une reconnaissance sur le plan national. En interne, ces pratiques peuvent être facilement transférées à d'autres pays. Par contre, le système des NVQ suppose un engagement de l'État et des branches industrielles qui est beaucoup plus complexe à mettre en place dans de nombreux pays. Les PME auraient pourtant beaucoup à gagner dans ce genre de système : facilité à identifier les compétences lors d'un recrutement, moindre hésitation des salariés à commencer leur carrière en PME, reconversions plus aisées en cas de difficultés de l'entreprise, etc.

19. En France, l'employeur est obligé légalement de dépenser en formation un pourcentage de la masse salariale. 
Dans la plupart des cas, on peut parler «d'organisations apprenantes », mais encore assez peu «d'organisations qualifiantes » pour les salariés ;

- à côté des formations traditionnelles «sur le tas» ou en «doublon» avec un ancien, on voit se développer dans certains cas l'auto-apprentissage avec des logiciels placés sur des ordinateurs proches du poste de travail avec le soutien de l'encadrement. Ces innovations montrent que les PME ont tout intérêt à concevoir des systèmes de formation souples, informels, proches de l'activité quotidienne, car les bénéfices tirés et l'effet d'engagement des salariés sont importants. De même, ces pratiques permettent de faire évoluer les salariés tout en étant compatibles avec une vision stratégique de l'avenir très limitée pour l'entreprise. Cependant, l'éloignement des cours «ex cathedra » et des dispositifs de formation plus formalisés, bien que plus adaptés aux PME, présente certains inconvénients. Pour les salariés, la formation n'est plus une garantie de progression dans une carrière ; elle devient seulement un moyen de conserver son poste, de maintenir son «employabilité ». Des évolutions très importantes en matière de compétences peuvent rester invisibles du fait de ces pratiques de formation diffuses. Aussi, les salariés auraient-ils intérêt à être attentifs à ces pratiques qui peuvent passer inaperçues ou bien à exiger des dispositifs de reconnaissance nouveaux tenant compte de ces constats. Leur motivation à poursuivre dans cette voie est en jeu et intéresse aussi l'entreprise.

\section{Bibliographie}

ANDREASEN, L.E., B. CORIAT, F.D. HERTOG et R. KAPLINSKY (éd.), (1995), Europe's Next Step. Organisational Innovation, Competition and Employment, Ilford, Frank Cass.

BAGNASCO, A. et C. SABEL (éd.), (1994), PME et développement économique en Europe, Paris, La Découverte.

BEL, M. et R. TORTAJADA (éd.), (1992), Europe, formation et citoyenneté d'entreprise, Paris, Minerve.

BERGERON, P.O. et M.A. GAIFFE (éd.), (1994), Croissance, compétitivité, emploi, Bruxelles, Presses interuniversitaires européennes.

CEDEFOP (1987), «Les petites et moyennes entreprises : un terrain de choix pour la formation professionnelle », Formation professionnelle, $\mathrm{n}^{\circ} 3$.

CEDEFOP (1988), «Le rôle des partenaires sociaux dans la formation professionnelle initiale et continue dans la CEE (rapport de synthèse)», Berlin.

CEE/Education Training Youth (1991), « Vocational training in the European community. Challenges and future outlook», Suivi du mémorandum de la Commission sur la formation technique dans la Communauté européenne des années 1990, Études $n^{\circ} 3$.

CEDEFOP (1992), «Le rôle de l'état et des partenaires sociaux : mécanismes et sphères d'influence », Formation professionnelle, $\mathrm{n}^{\circ} 1$. 
CEE, DGV (1994), «Observatoire de l'emploi. Tableau de bord », $\mathrm{n}^{\circ} 2$.

Directive 92/5/CEE du 10 février 1992 relative aux problèmes sanitaires en matière d'échanges intracommunautaires de produits à base de viande, Journal officiel, $\mathrm{n}^{\circ}$ L57 du 2 mars 1992.

Directive 93/43/CEE du 14 juin 1993 sur l'hygiène des denrées alimentaires, Journal officiel, $\mathrm{n}^{\circ}$ L175/1 du 19 juillet 1993, p. 399-409.

LUTZ, B. (1984), «Problèmes de formation professionnelle dans les petites et moyennes entreprises », Actualités de la formation permanente, $\mathrm{n}^{\circ}$ 67, p. 41-47.

SARNIN, P. (1990), «Changements stratégiques et pratiques de formation dans 16 PMI de Rhône-Alpes », Contribution au Colloque TETRA : «La PME, objet de recherche pertinent?», 30-31 mai 1990.

SERRES, M. (1992), Le Tiers-Instruit, Paris, Gallimard. 